\title{
Driven to it
}

\section{Trudie Roberts}

Like many medical women of my generation, I married later in life and so, owing more to inertia than principle, I never took up my married name. Consequently, not everyone knows or suspects that my surgeon husband and I are partners, particularly as we work on different sides of the Pennines. Some years ago, he was attending a specialty meeting and a colleague who happened to work on my side of the hills remarked, in a conversation regarding the teaching of medical students, 'You're lucky; we've got that left-wing feminist Professor $X$ telling us what to do!' ... meaning me! On returning home, my husband regaled me with this story and I was suitably flattered, not only because I'd made an impression, but also because I'd always considered myself a wet liberal who secretly subscribed to The Spectator. My faux aspirations to the sisterhood were soon punctured when I proudly retold this anecdote to my more radical female colleagues. Here I was met with such disparaging comments as, 'You a feminist: that's a laugh, you think Annie Oakley is a character out of a musical' and 'We've seen you buying the Daily Telegraph'. True, but I have read three chapters of The Female Eunuch and they had sold out of The Guardian, honest.

So perhaps my feminist credentials are not obvious to all. It is true that until relatively recently, I've never really thought about sexism in medicine. Over the years from medical student to consultant-hood there have been very few occasions, it seemed to me, when I have experienced behaviour that could be construed as misogynistic: being called 'dear' by a red-faced lascivious pathologist and surgical registrars referring to colleagues who were not working the 'one in twos' the rest of us were as doing the 'housewife shift'. All in all, either I've been rather impervious to such comments or perhaps there weren't a lot of them made in the environments I worked in. I had heard stories of how, in the past, female residents were once not allowed to eat in the doctors' dining room, but by the 1980s those seemed Dickensian tales. As now, however, the working environment was dominated by male consultants with female consultants in the minority. Most of the senior figures in medicine both locally and nationally are men, although in more recent years, a few women have achieved top positions in the Medical Royal Colleges, including the Royal College of Physicians.

I've drifted into middle age having achieved most of the things I desired and thought I was capable of without feeling that I have been done down by my gender. My surprise at hearing about the 'problem' of the feminisation of medicine has therefore probably been greater than that of my more perspicacious female colleagues.

Trudie Roberts, professor of medical education and honorary physician, University of Leeds
The history of women in medicine is mired in discrimination. The control of entry into medical studies by medical professionals went unchallenged for centuries. Attempts at entry by women in the 19th century illustrate the deep resistance of the male-dominated profession to opening its doors to 'others'. Educational and professional opportunities for women in the 19 th century were limited by social convention. As the century progressed, however, many women began to challenge these views. Educational pioneers (led by Emily Davies, founder of Girton College, Cambridge $)^{1}$ saw education as a remedy for women's lack of progress and rights, and colleges that admitted women were founded.

Nevertheless, a medical degree proved more difficult to access than an arts education because training required the cooperation of existing practitioners (all men). Some determined women, such as Elizabeth Blackwell (trained at St Bartholomew's, 1850), the first fully qualified woman doctor of medicine, and Elizabeth Garrett Anderson (trained at The London, 1863), the first English woman to qualify, championed medical education for women. ${ }^{2}$ Despite their individual success, there was still strong opposition to women entering medicine as Victorian society and religious ideals dictated that middle- and upper-class women stayed at home, caring for their family. For example, Ellen Colborne registered to study at Barts in 1865; her presence caused such a stir among the male students that lectures were disrupted and a petition was raised against her, which most students signed. She was forced to withdraw and women were not admitted again to Barts until the 1950s. ${ }^{3}$ The London School of Medicine for Women (later the Royal Free) was founded in 1874 and was the first UK medical school to accept women as more than just exceptions.

In 1944, the Goodenough Committee recommended that up to one-fifth of medical students should be female ${ }^{4}$, and three years later, other medical schools in the UK started to accept women. In 1963, the University Central Council on Admissions (UCCA) showed that women constituted only $29 \%$ of applicants to medical school, with numbers varying from $8.5 \%$ at Cambridge to $38 \%$ at Sheffield and $61 \%$ at the Royal Free.

The Royal Commission on Medical Education in 1968 showed that some medical schools applied more stringent selection criteria to women than to men. ${ }^{5}$

Current fairer selection might well have increased the admission of women, but undoubtedly the greater number of women entering medical schools is largely attributable to the increasing proportion of female applicants, as indeed is the case for degree courses overall. 6

Girls are now more likely than before to take science 'A' levels and their performance in these subject is as good as, if not better than, that of boys. ${ }^{7}$ Women might also be attracted by the 
changing nature of medicine and by newer approaches to medical education: more emphasis on communication skills and the social aspects of understanding health, less didactic and more experiential teaching, and a more patient-centred approach.

Over the past four decades, the proportion of women in medicine and at medical school has increased dramatically. The percentage of female medical school applicants increased from $35 \%$ in 1977 to over $55 \%$ in $2000 .{ }^{5}$ A recent survey showed that, in fact, applications from women to study medicine had fallen from $59 \%$ in 2003 to $55.7 \%$ in 2008 , indicating that the attractiveness of medicine to women might well have peaked. Interestingly, the acceptance rate in 2008 was $48.4 \%$ and $47.8 \%$ of applicants for women and men, respectively; for women, this had fallen from a high of $60.9 \%$ in $2003 .{ }^{8}$

What do these figures actually mean for the gender composition of the profession? A study commissioned by the Royal College of Physicians predicted that women doctors will reach a majority sometime between 2017 and 2022. ${ }^{9}$

Why should this be a problem? If girls do better at ' $A$ ' level than boys and more of them apply to medical school, and if the admission process is fair and appropriate, isn't it correct that more girls should be admitted? Apparently not for some people. The 'feminisation of medicine', is being highlighted as a problem. This 'problematisation' of women in medicine seems to have gone so far that within the past year I have seen official papers calling for quotas for admitting female students to medical schools. This is not only illegal under current equality and diversity legislation, but strikes at the heart of social justice and equity in our society. It suggests that the medical profession might be out of step with the rest of British society in its attitude towards women.

In 2008, the British Medical Journal published a debate entitled 'Are there too many female medical graduates?.'10,11 The main issues of debate cluster in two areas: effects on service delivery (workforce issues) and long-term effects on the status of the medical profession. The flames of this debate are being fanned by various myths and misapprehensions. Therefore, I will examine rationally some of these arguments, taking away the emotion and vehemence with which they are often infused. The most common assertion of the problem of having a majority of women in medicine is the collapse of the current healthcare service because women have children, work parttime and only choose family-friendly specialties. I will explore these issues in the next section. The debate about 'excessive' numbers of female doctors and their effect on the status of the medical profession is a more fundamental discussion about the identity of doctors and the medical profession. I will leave this for another time as it tends to generate more heat than light.

\section{The healthcare delivery argument}

Some assert that all women want to have babies and consequently want to work part-time and that, as a result, the health service as we presently know it could not be delivered and would collapse. Although it is true that many women (and men) want to have children, it is not a universal aim. Many childless women are not sad or unfulfilled but have made a choice that is right for them, a decision that is not necessarily based solely on their decision to have a career. There are days when, as a parent myself, I completely understand their decision.

McKinstry contends that increasing the number of women will ultimately affect the way in which healthcare is organised and delivered. ${ }^{11} \mathrm{He}$ argues that women concentrate on familyfriendly specialties, for example primary care and psychiatry, and tend not to take up the more time-commitment intensive specialties such as surgery. However, the leading specialty for women ( $40 \%$ women consultants) is paediatrics, which requires the extensive on-call and out-of-hours commitment that women are supposed to shun. Second, McKinstry ${ }^{11}$ bemoans that female doctors are more likely to work part-time, even after their children are independent. ${ }^{12}$ Scant regard is paid to emerging evidence that younger male doctors are also increasingly seeking part-time careers and a more flexible lifestyle. ${ }^{13}$ In a survey of attitudes to working conditions, over $72 \%$ of responding registrars 'would prefer, given no increase in current pay, to work 48 hours a week or less and even if pay was increased, only $8.4 \%$ would want to work more than 56 hours.'. ${ }^{14}$

Some women might take time off to have children, but they retire later and so stay in the workforce for longer. ${ }^{15}$ Furthermore, the implementation of the European Working Time Directive (EWTD) - which requires a 48-hour working week for all doctors and the widespread adoption of shift patterns of working in hospitals - means that the delivery of healthcare will change radically, regardless of the composition of the workforce.

To lay the blame for changing healthcare delivery solely at the door of 'feminisation' is a misleading argument. Underlying this debate is a much broader question: what sort of medical workforce do we need? The answer goes far beyond gender. A workforce that understands the demands of 'normal' life and is not chronically exhausted by a working week of 120 hours is to be applauded and not decried. Working 120 hours per week was not a golden age for anyone of any gender and certainly not for the patients we attended.

The second workforce issue used to increase moral panic over 'excessive' numbers of female entrants is the suggestion that there might be differences in activity rates (how many patients are seen in a session) between male and female doctors. ${ }^{16}$ This argument is often based on essentialist assumptions that women have different qualities and abilities and might work in different ways to men. Studies of patients' perceptions of male and female doctors demonstrate that medical encounters are frequently shaped by gender expectations and stereotypes. For example, one US study showed that patients felt more disappointment if their female doctor was not as approachable or warm as expected than if their male doctor had the same shortcoming. ${ }^{17}$ Another study revealed that patients who preferred a male doctor believed that males were more technically competent, whereas those who preferred female doctors believed that females expressed more humanistic behaviours. ${ }^{18}$ In this sense, hidden rules for the appropriate behaviour, treatment and appearance of female doctors seem to be as embedded in medical culture as they are in 
society generally. Kilminster et al reviewed the evidence that men and women practice medicine differently; ${ }^{19}$ the results were conflicting, not only in regard to how the two genders made treatment decisions but also in areas related to personal health and how male and female doctors respond to the psychological stresses of medical practice. ${ }^{20}$

Bloor et al examined Hospital Episode Statistics for England which showed that male doctors have significantly higher activity rates than women doctors even after accounting for age, specialty and place of work. ${ }^{16}$ They emphasized that this could have serious implications, with an increasingly female workforce leading to reduced productivity over time by reducing the throughput of patient numbers. The limitations of the study are, however, multiple. It is not universally agreed that 'activity rates' are the best measure of good clinical practice. Activity rates measure numbers of encounters rather than qualitative improvement resulting from the doctor-patient encounter and they could be gender-biased. Furthermore, this study did not look at health outcomes, patient satisfaction rates, patient compliance or likelihood for re-referral, neither was it able to take into account the size of the teams led by men or women, which would directly affect the number of patients seen under that consultant's name.

More importantly, all of this debate focuses on the past; the way in which healthcare is delivered in this country in the future is going to change radically anyway. Society already demands and expects healthcare delivery to be increasingly centred on its needs and wants, and this is what the NHS should focus on. This will increasingly mean that patients will seek consultations at times suiting their lifestyles, such as in the evenings and at weekends, and not at times that mean they need to take time off work or make special arrangements for child or dependent care. The increasing use of the internet to supplement face-to-face consultations will mean that doctors' working environment might be their home. Women have long provided the workforce for these types of non-standard work patterns, for example in retail. Even within the health service, the majority of this shift working has until recently been provided by nurses, a predominantly female workforce.

What conclusions can one draw? First, debates centring on gender and the practice of medicine, and the delivery of healthcare are not helped by emotional and evidence-free opinion. The issue for debate within influential organisations such as the Royal College of Physicians should not be the gender balance or imbalance, but how we can ensure that society gets the best doctors and a service that addresses its needs and expectations. This should not, and does not, depend on the proportion of women within the medical profession.

Finally, what of my standing as a 'radical feminist' - probably overstated, but in the future who knows? As a female colleague said to me after being asked to take the minutes of a meeting because she was the only woman present, 'It drives you to it...'

\section{Acknowledgement}

Some of the statistical and historical detail in this paper formed part of a publication on widening access to medicine written by myself and my colleague Dr Katharine Boursicot (Boursicot K, Roberts TE. Widening participation in medical education: challenging elitism and exclusion. Higher Education Policy 2009;22:19-36.).

\section{References}

1 David M. The state, family and education. London: Routledge, 1980.

2 St John Fancourt M. They dared to be doctors: Elizabeth Blackwell, Elizabeth Garrett Anderson. London: Longmans, 1965.

3 Waddington K. Medical education and St Bartholomew's Hospital, 1123-1995. London: The Boydell Press, 2003.

4 Goodenough Committee. Report of the interdepartmental committee on medical schools. London: HMSO, 1944.

5 McManus IC. Medical school applications - a critical situation. BMJ 2002;325:786-7.

6 Lurie N. Preventative care for women: does sex of the physician matter? N Engl J Med 1993;329:478-82.

7 Grant J, Jones $\mathrm{H}$, Lambert T. An analysis of trends in applications to medical school. Milton Keynes, Oxford: Open University Centre for Education in Medicine/UK Medical Careers Research Group, 2002.

8 British Medical Association. Equality and diversity in UK medical schools. London, BMA: 2009. http://www.bma.org.uk/images/ eocreport_tcm41-192128.pdf

9 Elston M. Women in medicine. London: Royal College of Physicians, 2009.

10 Dacre J. Are there too many female medical graduates? No. BMJ 2008;336:749.

11 McKinstry B. Are there too many female medical graduates? Yes. BMJ 2008;336:748.

12 Gravelle H, Hole AR. The work hours of GPs: survey of English GPs. Br J Gen Pract 2007;57:96-100.

13 Mather HM. Specialist registrars' plans for working part-time as consultants in medical specialties: questionnaire study. $B M J$ 2001;322:1578-9.

14 Goddard AF, Evans T, Phillips C. Medical registrars in 2010: experience and expectations of the future consultants of the UK. Clin $\mathrm{Med}$ 2011;11:532-5.

15 Batchelor AJ. Senior women physicians: the question of retirement. $N$ Y State J Med 1990;90:292-4.

16 Bloor K, Freemantle N, Maynard A. Gender and variation in activity rates of hospital consultants. J R Soc Med 2008;101:27-33.

17 Hall J, Irish J, Roter D et al. Gender in medical encounters: an analysis of physician and patient communication in a primary care setting. Health Psychol 1994;13:385-92.

18 Fennema K, Meyer DL, Owen N. Sex of physician: patients' preferences and stereotypes. J Family Pract 1990;30:441-6.

19 Kilminster S, Downes J, Gough B et al. Women in medicine - is there a problem? A literature review of the changing gender composition, structures and occupational cultures in medicine. Med Educ 2007;41:39-49.

20 Toews J, Lockyer J, Dobson D. Analysis of stress levels among medical students, residents, and graduate students at four Canadian schools of medicine. Acad Med 1997;72:997-1002.

Address for correspondence: Prof Trudie Roberts, Leeds Institute of Medical Education, University of Leeds, Room 7.09, Level 7, Worsley Building, Leeds, LS2 9NL. Email: T.E.Roberts@leeds.ac.uk 